\title{
Study on the creep and fatigue interaction of a Nimonic Alloy 75 under multiaxial nonproportional loading
}

\author{
Zhilan Zhan ${ }^{1, a}$ and Gaoping Liü, ${ }^{1 *}$ \\ ${ }^{1}$ School of Mechanical Engineering and Automation, Wuhan Textile University, No.1 Fangzhi Road, \\ Luxiang, Wuhan, P. R. China 430073 \\ a1990007@wtu.edu.cn \\ bliugaoping_007@126.com
}

Keywords: Unified viscoplastic model. Elevated temperature. Nimonic alloy 75. Multiaxial non-proportional cyclic.

Abstract. In this paper, a unified viscoplastic model was utilised to numerically simulate cyclic hardening of a nimonic alloy 75 under multiaxial non-proportional cyclic straining at elevated temperature. Description of the constitutive model and interpretation of material parameter were also presented. Constitutive simulations for the circular and square cycling strain paths in the axial-torsional plane have been verified by the test results, and the possibility of this model extension was also discussed.

\section{Introduction}

Understanding multiaxial cyclic deformation behaviors is important in inelastic structural analysis including fatigue assessment of structural components in the industry since structural components are subject to complex stressings or strainings in practical [1]. Nonproportional cyclic loading causes more damage than proportional loading and occasionally reduces low cyclie fatigue. During the analysis of components subject to multiaxial load, the problem often goes to an "equivalent" uniaxial fatigue case without thought as to whether the simplifying assumptions are valid for the specific load sequence or component being considered. Although in recent years, amount of research work on this area have been published, however, for this non-expert field, these works often are difficult to digest and complex to apply [2].

Over last several decades, a number of constitutive models describing inelastic deformation for the multiaxial loading at elevated temperature have been proposed [3-9]. But less published researches on the creep and fatigue problem under multiaxial non-proportional strain and stress cycling loading are conducted. So development of more general and proper constitutive models may be a root to solve above problems and difficulties of the multiaxial fatigue. This kind of constitutive models can be used to simulate more general and complex loading by the incremental plasticity and viscoplasticity procedures, and also can be finally implemented into the structure analysis and design software like ABUQUS and ANSYS et al. for industry practice purpose.

This paper presents a primarily modelling of the creep and fatigue interaction under multiaxial strain loading. The plasticity and viscoplasticity constitutive model include non-linear kinematic hardening rule was proposed by Chaboche [9]. The main work focus upon the constitutive simulation of a nimonic alloy 75 under multiaxial non-proportional cyclic straining conditions for verifying the model application.

\section{Experimental description}

The experimental results were obtained in a Nimonic Alloy 75. An MTS809 servo-hydraulic testing machine was used with the computer controlled loading spectra programmed as required. An electric resistance furnace was used with the temperature controlled at $620 \pm 5^{\circ} \mathrm{C}$. An extensometer 
$(25+2.5 /-2.5 \mathrm{~mm})$ was used to monitor the strain. All tests were carried out under strain control at a constant strain rate $0.2 \% / \mathrm{s}$

Smooth specimens with a diameter of $16 \mathrm{~mm}$ and gauge length of $30 \mathrm{~mm}$ were used for the uniaxial experiments and parameters estimated. A baseline cyclic test and a multiple-step cyclic test were carried out to obtain the full cyclic stress-strain. A strain ratio $R_{\varepsilon}=-1$ and the strain range of $2 \%$ were used for the baseline test while strain ranges of $1.2,1.6$ and $2 \%$ were used for the multiple-step test at $R_{\varepsilon}=-1$. Multiple hardening and relaxation test were carried out where the strain was held constant at selected levels $(1.0 \%-2.2 \%)$ for $100-300$ seconds. The stress relaxation was recorded during the tests.

Specimens employed for multiaxial experiments were thin-walled tube with the outside diameter, wall thickness and gage length of $15 \pm 0.05,3 \pm 0.05$ and $100 \mathrm{~mm}$, respectively. And the axial strain range $\Delta \varepsilon / 2$ and torsional strain range $\Delta\left(\gamma / 3^{1 / 2} / 2\right)$ are $0.8 \%$. The strain cycling path includes circular and square strain loading for the multiaxial nonproportional cyclic hardening were used here for the constitutive simulation purpose. The effective stress $|\hat{\sigma}|$ is denoted as the effective stress amplitude (with $\Delta \sigma / 2$ the axial stress range and torsional stress range):

$$
\Delta \sigma_{M} / 2=\left[(\Delta \sigma / 2)^{2}+(\Delta(\sqrt{3} \tau) / 2)^{2}\right]^{1 / 2}
$$

\section{Chaboche Constitutive Equations}

The multiaxial constitutive equations utilized here are based on Chaboche model [9], which is listed at Appendix I. In this formulation the material parameters of the model $Z, n, C_{1}, C_{2}, C_{3}, a_{1}, a_{2}, a_{3}, b, Q, k, b_{1}, \Phi_{\infty}$ are all temperature-dependent. The kinematic hardening parameters $a_{1}, a_{2}, a_{3}$ and $C_{1}, C_{2}, C_{3}$, isotropic hardening parameters $Q, b$ and kinematic recovery parameters $b_{1}$ and $\Phi_{\infty}$, the viscoplasticity parameters of time-dependent $n, Z, k$. The method of determining these parameters based on the unaxial experimental data, has been discribed in previous work [10, 11 and 12]. The values of material parameter in the constitutive model are listed in Table 1.

Table 1. Materials parameters estimated

\begin{tabular}{ll}
\hline Material parameters & Values of parameters \\
\hline $\mathrm{E}(\mathrm{MPa})$ & 145 \\
$\mathrm{G}(\mathrm{MPa})$ & 55 \\
$\mathrm{~b}$ & 7.8 \\
$\mathrm{Q}(\mathrm{MPa})$ & 136 \\
$\mathrm{a}_{1}(\mathrm{MPa})$ & 121 \\
$\mathrm{C}_{1}$ & 632 \\
$\mathrm{a}_{2}(\mathrm{MPa})$ & 85 \\
$\mathrm{C}_{2}$ & 459 \\
$\mathrm{a}_{3}(\mathrm{MPa})$ & 36 \\
$\mathrm{C}_{3}$ & 287 \\
$\mathrm{Z}\left(\mathrm{MPaS}{ }^{1 / \mathrm{n}}\right)$ & 371 \\
$\mathrm{n}$ & 17 \\
$\mathrm{~K}(\mathrm{MPa})$ & 110 \\
$\Phi_{\infty}$ & 0.78 \\
\hline
\end{tabular}

\section{Simulation and Discussion}

The constitutive simulation program [13, 14, and 15] of multiaxial nonproportional loading (strain controlled) were modified on the present model. Comparisons of experimental and simulated results of the effective stress amplitudes for two nonproportional strain cycling path are shown in the Fig.1. Fig.1 (a) presents experimental and simulated results of effective stress responses of a circular strain path for 
number of cycles $\mathrm{N}=1-50$ with constant effective strain amplitude $0.8 \%$ and a constant strain rate $0.2 \% / \mathrm{s}$. Fig. 1 (b) presents experimental and simulated results of effective stress responses of a square strain path for number of cycles $\mathrm{N}=1-50$ with constant effective strain amplitude $0.8 \%$ and a constant strain rate $0.2 \% / \mathrm{s}$.

In Fig.1, the experimental results present the rapid reduction of stress amplitude width for describing the cyclic hardening behaviour. The cyclic stabilization followed by, can also be seen during initial few cycles $(\mathrm{N}=1-10)$ in the Fig.1. Good simulation on the effective stress responses can be observed under both of circular and square paths in Fig.1 (a) and (b), although some over-estimates exist especially for the square strain path. The simulation results are still encouraging; the capability of the present constitutive model is verified by comparisons between simulation and experimental results.

According to the above comparisons, one reason of over-estimate may be caused by the material parameter estimates; the optimisation procedure of material parameters is not carried out in this paper, another one may be that the additional hardening of non-proportional loading may need to be considered in the modelling. In generally, the effect of creep on cyclic plastic behaviours comes to be impartment, especially, at the high temperature. Under the square stress path with the trapezoidal strain cycles, the axial stress and torsional stress holding alternate, the creep and fatigue interaction may be an influence on the experimental results. So the present model without considering creep and fatigue interaction effect may cause the more over-estimate simulation of experimental result than simulation of the circular stress path. Hence, more effects of creep and fatigue interaction on the constitutive model should be incorporated into further studies.

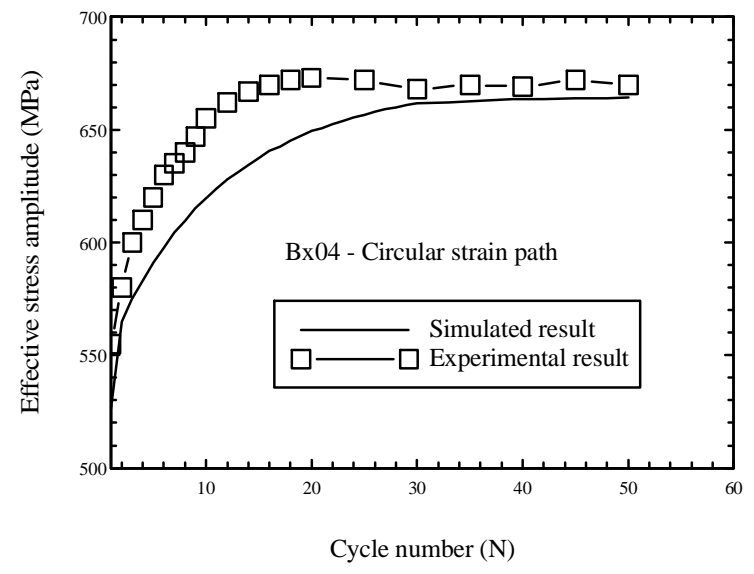

(a) Circular path

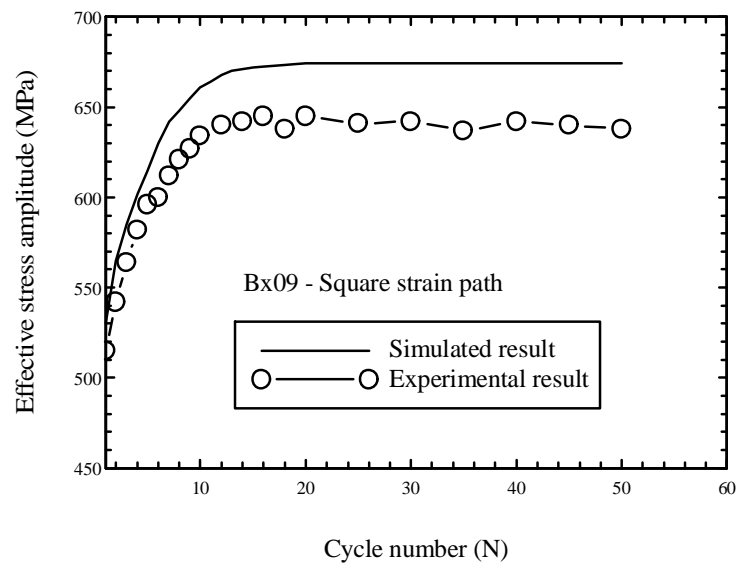

(b) Square path

Fig.1 Comparison of experimental result and simulated result of the effective stress amplitudes for two strain control path.

\section{Acknowledgments}

The authors are grateful to the Returning Overseas Scholar Funding of MOE ([2008] No.890) and the Science Research Grants (D20091706) of the Hubei Education Department and Dr. Funding of Wuhan Textile University for the financial supports. And also appreciate very much to Dr. Juan Zhang and Dr. Yujie Liu of Southwest Jiaotong University for the numerical program and experiments support

\section{References}

[1] Y. Ohashi, J., Eng. Mater. And Tech. 107(1985)101-109.

[2] D.F. Socie, , and G. B. Marquis, Multiaxial Fatigue, Society of Automotive Engineering, Inc., Warrendale, U. S. A., 2000. 
[3] J. Lemaitre and J. L. Chaboche, Mechanics of Solid Materials, Cambridge University Press, Cambridge, UK, 1990

[4] D. L. McDowell, J. Appl. Mech.54 (1987)323-334.

[5] N. Ohno, and J. D. Wang, Int. J. Plast. 9(1993) 375-401

[6] Y. Jiang, and H. Sehitoglu, J. Appl. Mech. 163(1996)720-725.

[7] E. Tanaka, European J. Mech., A/Solid. 13(1994)155-173.

[8] T. Itoh, X. Chen, T. Nakagawa and M. J. Sakane, Eng. Mater. And Tech.122 (2000)1-10.

[9] J. L. Chaboche, Int. J. Plasticity. 5(1989)247-302.

[10] Z. L. Zhan and J. Tong, Mech Mater. 2007, 39(2007)64-72.

[11] Z. L. Zhan, G. P. Liu and X. C. Chen, Modelling of cyclic plasticity and viscoplasticity of a nimonic alloy 75, Advanced Materials Research. 146-147(2011)1617-1620.

[12] Q. L. Zeng and Z. L. Zhan, The application of non-linear optimization algorithm to the simulation of nimonic alloy 75 at elevated temperature, Advanced Materials Research. 1061-1062(2015)39-44.

[13] Kang, G.Z., Commu. Numer. Mech. Engn., 22(2006)137-153.

[14] Abdel-Karim, M., Int. J. Plast. 21(2005)1303-1321.

[15] Abdel-Karim, M. and Ohno N., Int. J. Plast. 16(2000)225-240.

\section{Appendix I: The Chaboche unified model adopted in this work}

Yield Surface

$$
\begin{aligned}
& f=J(\hat{\sigma}-\hat{\chi})-R-k \leq 0 \\
& J(\hat{\sigma}-\hat{\chi})=\sqrt{3 / 2\left(\hat{\sigma}^{\prime}-\hat{\chi}^{\prime}\right):\left(\hat{\sigma}^{\prime}-\hat{\chi}^{\prime}\right)}=\sqrt{3 / 2}|| \sigma^{\prime}-\chi^{\prime} \|
\end{aligned}
$$

Kinematic Hardening

$$
\begin{aligned}
& \hat{\chi}=\sum_{i=1}^{m} \hat{\chi} \\
& \chi_{i}^{夭}=C_{i}\left(a_{i} \varepsilon_{p}-\hat{\chi}_{i} \Phi \Phi_{\infty} \quad i=1-3\right. \\
& \Phi=\Phi_{\infty}+\left(1-\Phi_{\infty}\right) e^{-b_{1} P}
\end{aligned}
$$

Isotropic Hardening $\quad \delta=b(Q-R)$

Flow Rule

$$
\begin{aligned}
& \varepsilon_{p}^{\&}=\frac{3}{2}\left\langle\frac{J(\hat{\sigma}-\hat{\chi})-R-k}{Z}\right\rangle^{n} \frac{\hat{\sigma}^{\prime}-\hat{\chi}^{\prime}}{J(\hat{\sigma}-\hat{\chi})} \\
& \langle x\rangle=x \mathrm{H}(x) \quad \mathrm{H}(x)= \begin{cases}1, & x \geq 0 \\
0, & x<0\end{cases}
\end{aligned}
$$

Accumulated Plastic Strain

$$
\delta=\sqrt{(2 / 3) \varepsilon_{p}^{\&}: \varepsilon_{p}^{\&}}
$$

Parameters to be identified: $Z, n, C_{1}, C_{2}, C_{3}, a_{1}, a_{2}, a_{3}, b, Q, k, b_{1}, \Phi_{\infty}$ 